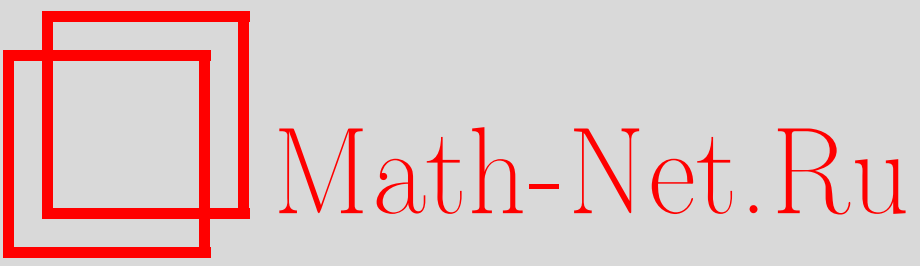

А. П. Соколов, В. Н. Щетинин, М. Ю. Козлов, Моделирование упругих свойств композитных материалов методом асимптотического осреднения с учетом неидеального интерфейса компонент, Матем. моделирование, 2020, том 32 , номер 8, 119-138

DOI: https://doi.org/10.20948/mm-2020-08-08

Использование Общероссийского математического портала Math-Net.Ru подразумевает, что вы прочитали и согласны с пользовательским соглашением

http://www.mathnet.ru/rus/agreement

Параметры загрузки:

IP : 54.198 .64 .247

26 апреля 2023 г., 10:16:57 


\title{
МОДЕЛИРОВАНИЕ УПРУГИХ СВОЙСТВ \\ КОМПОЗИТНЫХ МАТЕРИАЛОВ \\ МЕТОДОМ АСИМПТОТИЧЕСКОГО ОСРЕДНЕНИЯ \\ С УЧЕТОМ НЕИДЕАЛЬНОГО ИНТЕРФЕЙСА КОМПОНЕНТ
}

\author{
(C) 2020 2. А.П. Соколов ${ }^{1}$, В.Н. Щетинин ${ }^{2}$, М.Ю. Козлов ${ }^{3}$
}

МГТУ им. Н.Э. Баумана

11alsokolo@bmstu.ru, 22sch_vitaliy@mail.ru, ${ }^{3}$ kozlovmy@student.bmstu.ru

DOI: $10.20948 / \mathrm{mm}-2020-08-08$

Представлена модификация метода асимптотического осреднения для решения задачи гомогенизации упругих свойств композитных материалов с учетом упругости интерфейса между фазами. Рассматриваются условия мягкого неидеального интерфейса, допускающие скачок поля перемещений при переходе через границу фаз. Предложен переход от модели композита с тонким межфазным слоем к модели с неидеальным интерфейсом. Представлен литературный обзор методов моделирования интерфейса между матрицей и наполнителем. Для численной реализации метода осреднения используется метод конечных элементов. Предложена модель поверхностного интерфейсного конечного элемента, реализующего скачок поля перемещений при переходе через границу фаз. Численный метод осреднения упругих свойств адаптируется к наличию разрыва поля перемещений. Оцениваются пороговые значения относительной жесткости и толщины межфазного слоя, для которых применимо моделирование с помощью условий скачка перемещений. Ставится задача идентификации параметров интерфейса по экспериментально полученным техническим константам композита. Вычислительные эксперименты проводятся для дисперсно-армированного и однонаправленного композита с изотропным включением.

Ключевые слова: композитные материалы; метод гомогенизации; метод асимптотического осреднения; эффективные свойства композитов; неидеальный интерфейс; метод конечных элементов; интерфейсный конечный элемент; адгезионный слой; параметрическая идентификация.

\section{MODELING ELASTIC PROPERTIES OF COMPOSITES USING ASYMPTOTIC AVERAGING METHOD WITH IMPERFECT INTERFACE}

\author{
A.P. Sokolov ${ }^{1}$, V.N. Shchetinin ${ }^{2}$, M.Yu. Kozlov ${ }^{3}$ \\ Bauman MSTU \\ 1'alsokolo@bmstu.ru, 2.sch_vitaliy@mail.ru, 33 kozlovmy@student.bmstu.ru
}

The paper presents a modification of the asymptotic averaging method for solving the 
elastic properties homogenization problem for composite materials, taking into account the phases interface elasticity. The conditions of a soft imperfect interface are considered, which allow a displacements jump on phases boundary. A literature review of methods for the interface modeling in composite materials is presented. The finite element method is used for the numerical implementation of an averaging method. A model of a surface interface finite element is proposed. The numerical method of elastic properties averaging is adapted to the presence of a discontinuity in the displacement field. Application boundaries of the soft imperfect interface are estimated in terms of interphase layer properties ranges. The problem of interface parameters identification by experimental data is considered. Computational experiments are conducted for dispersed-reinforced and unidirectional composite with isotropic inclusion.

Keywords: composite materials; homogenization; asymptotic averaging method; effective properties of composites; imperfect interface; soft imperfect interface; finite element method; interface finite element; interphase layer; adhesion; parametric identification.

\section{1. Введение}

На сегодняшний день к точности математических моделей композитных материалов (КМ) предъявляются все более высокие требования. Одним из перспективных направлений развития является разработка моделей композитов с учетом механики адгезии между матрицей и армирующим материалом. Адгезией принято называть поверхностное явление установления механической связи между телами. Экспериментально установлено, что в результате сложных физико-химических явлений на поверхности контакта фаз, протекающих в процессе технологического производства композитного материала, между матрицей и армирующим материалом возникает тонкий слой (адгезионный слой), обладающий отличными от них механическими свойствами.

Как правило, при моделировании КМ используют ту или иную теорию эффективных сред (теорию гомогенизации), задачей которой является построение процедуры перехода от структурно неоднородных сред (композитов) к однородным с осредненными (эффективными) свойствами. В классических подходах к расчету эффективных свойств для связи полей перемещений и напряжений в матрице и наполнителе используют условия идеального интерфейса. Данные условия постулируют неразрывность перемещений и нормальных напряжений при переходе через границу фаз, исходя из гипотезы идеальной связи и условий локального равновесия соответственно. При расчете упругих осредненных свойств учет того, что фазы взаимодействуют не напрямую, а через тонкую упругую пленку, необходим для правильной оценки вклада свойств армирующей фазы в свойства композита в целом [1].

Помимо естественного физико-химического соединения фаз на адге- 
зию могут оказывать влияние два фактора: поверхность контакта подвержена появлению механических дефектов производства, связанных с усадкой и/или проблемами диффузии связующего; для улучшения прочностных свойств применяют специальные технологические покрытия армирующего, к примеру, составы с нанотрубками.

Существует два подхода к моделированию адгезии. В первом отталкиваются от детального моделирования физико-химических процессов установления механической связи $[2,3]$. Например, в [4] упругие свойства адгезионного слоя оценивали с помощью методов молекулярной физики, основываясь на гипотезах о молекулярном строении межфазного слоя. Были получены аналитические зависимости упругих констант от молекулярных и структурных характеристик слоя. Во втором подходе отталкиваются не от причин появления адгезии, а от макроскопического эффекта, который вносит адгезия в напряженно-деформированное состояние композитного материала. Разрабатывают параметризованные модели интерфейса между телами, а параметры идентифицируют по макро-характеристикам композита. В рамках данного исследования будем пользоваться вторым подходом и приведем небольшую историческую справку о его развитии.

В ранних работах по данному направлению для моделирования упругого интерфейса между фазами вводили дополнительную виртуальную фазу - тонкую объемную пленку, покрывающую наполнитель. Такой подход получил название трехфазного моделирования (three-phase-material theory). Аналитические трехфазные модели рассмотрены в [5-8]. Интерфейсы между матрицей, слоем и наполнителем считаются идеальными. Численные расчеты эффективных свойств с помощью трехфазной модели проводили в более современной работе [9]. Данный подход обладает набором существенных недостатков: толщина межфазного слоя неизвестна и трудноизмерима; масштаб толщины на порядки меньше масштаба включений композита, что приводит к дополнительным трудностям в численном анализе.

Альтернативным подходом является исключение межфазного слоя из геометрического описания структуры материала в связи с малостью его толщины. При этом учет адгезии производится с помощью соотношений неидеального интерфейса на границах матрицы и армирующего материала. Модели неидеального интерфейса допускают разрывы полей перемещений и напряжений.

Работу Голланда и Рейснера [10] принято считать первой публикацией в данном направлении. Авторы предположили, что взаимодействие двух твердых тел с адгезионной связью можно рассчитывать с помощью введения бесконечного количества пружин, соединяющих поверхности данных 
тел. Такой интерфейс, получивший название интерфейса пружинного типа (spring type), вводит в расчет скачок поля перемещений на границе тел, пропорциональный величине нормальных напряжений в интерфейсе. Также в данной работе, исходя из физических соображений, было предложено считать поле нормальных напряжений на границе фаз непрерывным, если отношение жесткости интерфейса к жесткости тел мало.

В приложении к расчетам эффективных свойств композитных материалов такой подход был исследован в работах Бенвенисте $[7,11]$ и Хашина [12]. Работы Хашина [12-16] направлены на решение проблемы невозможности измерить свойства межфазного слоя напрямую. Его основная идея заключается в том, что если интерфейс оказывает существенное влияние на упругие свойства композита в целом, значит, его упругие свойства должны существенно отличаться от упругих свойств матрицы и армирующего, в частности, он должен быть существенно мягче. Такой тип интерфейса впоследствии назовут мягким интерфейсом (soft interface). Используя гипотезу о мягком интерфейсе, разложение в ряд Тейлора физических полей в области межфазного слоя и гипотезу об изотропности межфазного слоя, Хашин вывел эффективные упругие свойства и коэффициенты теплового расширения для дисперсно-армированного и однонаправленного волокнистого композита. Также в [14] Цви Хашин показал, как свойства неидеального интерфейса связаны со свойствами объемного межфазного слоя и его толщиной. Хашин и Бенвенисте независимо друг от друга обобщили классические вариационные принципы теории упругости для составных тел на случай неидеального интерфейса со скачком перемещений $[11,15]$. В [17] Питер Бовик успешно объединил разложение в ряд Тейлора с использованием поверхностных операторов дифференцирования, что позволило вывести соотношения неидеального интерфейса для произвольной геометрии армирующей фазы. В работе [18] Бенвенисте и Милош, объединяя идею Бовика с работой [19], показали, что межфазный слой высокой жесткости характеризует скачок нормальных напряжений при переходе через границу фаз. Такой интерфейс получил название жесткого интерфейса (stiff interface или hard interface). В [20] Бенвенисте, обобщая идеи мягкого и жестокого интерфейсов, вывел соотношения для трехмерного анизотропного тонкого слоя произвольной жесткости.

Альтернативный подход к выводу определяющих соотношений межфазного интерфейса представлен Андерсом Кларбрингом [21, 22]. Он предложил использовать метод асимптотического разложения в степенной ряд по малому параметру толщины межфазного слоя и вывел таким образом соотношения для мягкого интерфейса. Глубокую теоретическую проработку 
вопросов применимости данного подхода можно найти в [23]. Широкое развитие метод асимптотического разложения для механики интерфейсов получил в работах Фредерика Лебона, Рафаэллы Риццони, Серге Дюмонта: в $[24,25]$ подход обобщен на случай нелинейного поведения мягкого межфазного слоя; в [26] - на случай жесткого интерфейса; в [27] исследовано влияние высших степеней разложения.

Стоит отметить, что существует множество как аналитических, так и численных методов гомогенизации неоднородной среды. Выбор способа описания неидеального интерфейса, который вводится в процедуру осреднения, построенной на базе конкретной теории, является задачей для отдельного исследования. В частности, Цви Хашин в своих работах [12-16] пользовался аналитическим обобщенным методом самосогласования. В последнее время широкое развитие получили методы численной гомогенизации. Одним из подходов к построению численной процедуры расчета эффективных свойств композита является Метод асимптотического осреднения (МАО), разработанный Н.С. Бахваловым [28] для осреднения дифференциальных уравнений в частных производных и примененный Б.Е. Победрей [29] для решения задач гомогенизации свойств композитов. Теоретическое обоснование возможности использования условий неидеального интерфейса в МАО показана в работах [29-32]. Процедура осреднения в МАО построена на решении локальных задач на ячейке периодичности (ЯП) материала, представляющих собой набор дифференциальных уравнений с периодическими условиями. В работах Ю.И. Димитриенко и А.П. Соколова $[33,34]$ представлена численная реализация метода Н.С. Бахвалова и Б.Е. Победри, основанная на сведении задач на ЯП к задачам «классического типа». Получаемые задачи решались методом конечных элементов (МКЭ).

Для численного моделирования механики взаимодействия тел с учетом упругости интерфейса между ними используют специальные интерфейсные (иногда называют когезионными) конечные элементы. Идея использования интерфейсных элементов для моделирования как упругих, так и неупругих законов взаимодействия фаз композита представлена в [35]. Примеры использования конечных элементов из коммерческих программных продуктов для моделирования интерфейса между фазами можно найти в работах [36-38]. Наиболее подходящим для такого моделирования является элемент INTER204 из пакета ANSYS Mechanical APDL [39].

В данном исследовании авторы адаптируют численную реализацию MOA, представленную в [33], для расчета упругих эффективных свойств с условиями неидеального интерфейса. Используется модель мягкого межфазного слоя. Выводится математическая модель поверхностного интер- 
фейсного конечного элемента для реализации условий неидеального интерфейса при решении локальных задач. Программная реализация данного конечного элемента, разработанная авторами, доступна в рамках конечноэлементного фреймворка с открытым исходным кодом nla3d [40]. Представленный поверхностный интерфейсный элемент используется в программной реализации МАО. Проводится ряд численных экспериментов по сравнению результатов расчета упругих эффективных свойств при моделировании упругости взаимодействия фаз с помощью трехфазной модели и условий неидеального интерфейса для дисперсно-армированного и однонаправленного композита с изотропным включением. Представлены постановка и решение задачи идентификации параметров неидеального интерфейса по экспериментально полученным свойствам композита.

\section{2. Метод асимптотического осреднения с моделью интерфейса между фазами}

Рассмотрим расчетную область из композитного материала (рис.1а), состоящую из матрицы $m$, наполнителя $f$ и тонкого межфазного слоя $I$ с толщиной $D$. В работах Н.С. Бахвалова и Б.Е. Победри $[28,29]$ показано, как разложить систему уравнений линейной теории упругости для такой неоднородной среды на уравнения для однородной среды относительно осреднённых компонент тензора модулей упругости $\bar{C}_{i j k l}$, называемых эффективными, и на так называемые «локальные задачи» на ячейках периодичности (ЯП) (рис.1б) с периодическими граничными условиями и интегральным условием нормировки.

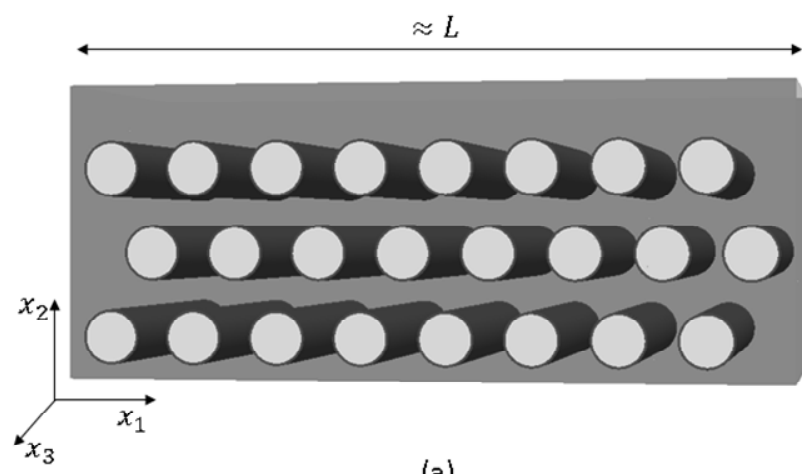

(a)

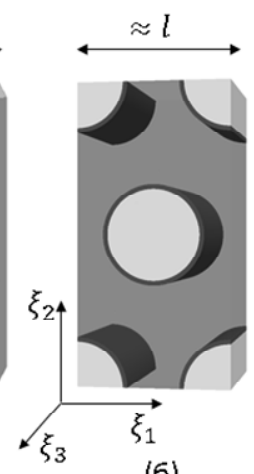

(6)

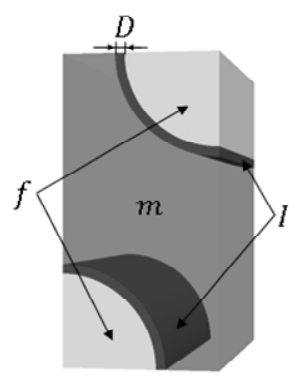

(B)

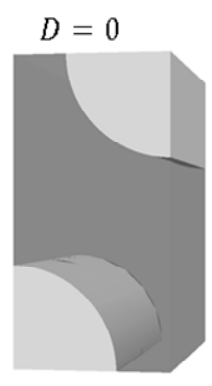

(r)

Рис.1. Расчетная область (а) неоднородной среды с межфазным слоем, (б) ячейки периодичности, (в) $1 / 8$ ячейки периодичности, (г) 1/8 ячейки периодичности для расчета с условиями неидеального интерфейса.

Согласно работе Ю.И. Димитриенко и А.П. Соколова [33], с учетом допущения об ортотропности композита и симметричности ЯП относительно 
боковых граней, локальные задачи можно свести к набору задач $L_{p q}$ с классическими краевыми условиями на 1/8 ЯП. Данные задачи при включении в рассмотрение межфазного слоя (рис.1в) можно записать так

$$
\left\{\begin{array}{l}
\frac{\partial \sigma_{i j(p q)}}{\partial \xi_{i}}=0 ; \\
\varepsilon_{i j(p q)}=\frac{1}{2}\left(\frac{\partial U_{i(p q)}}{\partial \xi_{j}}+\frac{\partial U_{j(p q)}}{\partial \xi_{i}}\right) ; \\
\sigma_{i j(p q)}=C_{i j k l}^{\alpha} \varepsilon_{i j(p q)} ; \quad \xi \in V_{\alpha} ; \quad \alpha=\{f, m, I\}, \\
{\left[U_{i(p q)}\right]=0 ; \quad\left[\tau_{i(p q)}\right]=0 ; \quad \xi \in \Sigma_{f I}, \Sigma_{I m},}
\end{array}\right.
$$

где $\sigma_{i j(p q)}, \varepsilon_{i j(p q)}$ - компоненты тензора «псевдонапряжений» и «псевдодеформаций», $U_{i(p q)}$ - компоненты вектора «псевдоперемещений» локальной задачи $L_{p q}, C_{i j k l}^{\alpha}$ - компоненты тензоров упругих модулей, где верхним индексом $\alpha=f, m, I$ обозначаются характеристики наполнителя, матрицы и межфазного слоя соответственно; $\xi=x_{i} / l$ - «быстрые координаты», изменяющиеся в объёме ячейки периодичности $V$. В системе присутствуют условия идеального интерфейса между компонентами, а именно отсутствие скачка векторов «псевдоперемещений» и нормальных «псевдонапряжений» $\tau_{i(p q)}=\sigma_{i j(p q)} n_{j}$ на поверхностях $\Sigma_{f I}, \Sigma_{I m}$ (рис.2a). Задачи $L_{p q}$ решают с граничными условиями классического вида на гранях ЯП и плоскостях симметрии, которые подробно описаны в [33].

Получившиеся задачи $L_{p q}$ обладают рядом существенных недостатков.

- Толщину слоя $D$ и компоненты тензора $C_{i j k l}^{I}$ трудно определить экспериментально, а подходы, основанные на детальном моделировании характеристик межфазного слоя, сильно зависят от типов исследуемых материалов и технологического процесса их производства.

- Толщина слоя $D$ обычно много меньше характерного размера ячейки периодичности $l$. Разрыв масштабов, присутствующий в задаче, усложняет численное моделирование.

Как было указано во введении, при условии малости толщины слоя $D \rightarrow 0$ его можно исключить из геометрии расчетной области (геометрии ЯП в нашем случае - рис.1г), а в математическую модель добавить соотношения, связывающие поля перемещений и нормальных напряжений на границе матрицы и наполнителя. Опираясь на общий вид условий неидеально- 
го интерфейса из [20], запишем выражения для скачка полей «псевдоперемещений» и «псевдонапряжений» при переходе через поверхность $\Sigma_{f m}$ как функции вида

$$
\begin{cases}{\left[U_{i(p q)}\right]=F\left(U_{i(p q)}^{f}, U_{i(p q)}^{m}, \tau_{i(p q)}^{f}, \tau_{i(p q)}^{m}\right) ;} & \xi \in \Sigma_{f m} \\ {\left[\tau_{i(p q)}\right]=G\left(U_{i(p q)}^{f}, U_{i(p q)}^{m}, \tau_{i(p q)}^{f}, \tau_{i(p q)}^{m}\right) ;} & \xi \in \Sigma_{f m},\end{cases}
$$

где $U_{i(p q)}^{f}, U_{i(p q)}^{m}, \tau_{i(p q)}^{f}, \tau_{i(p q)}^{m}-$ компоненты вектора «псевдоперемещений» и вектора нормальных «псевдонапряжений» со стороны фаз $f$ и $m$ соответственно (рис.2б).
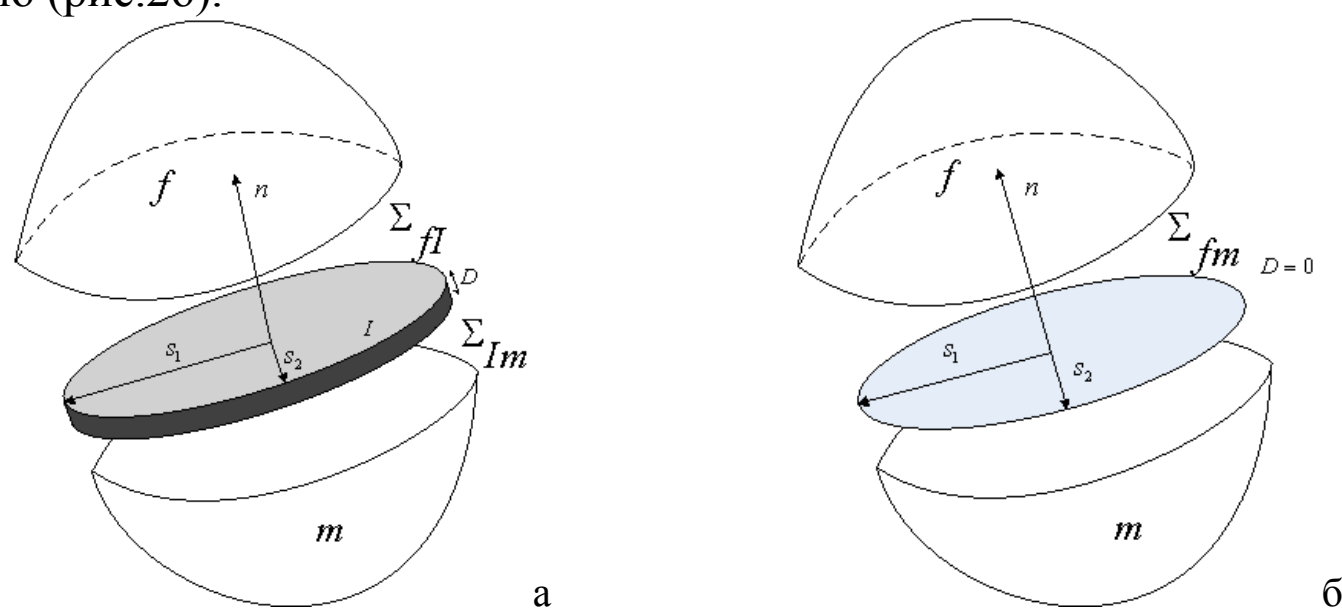

Рис.2. Трехфазная модель композита (а) и двухфазная модель композита с неидеальным интерфейсом на поверхности $\Sigma_{f m}($ б).

В [20] показано, если адгезионный слой предполагается изотропным и компоненты тензора упругих модулей адгезионного слоя предполагаются малыми по отношению к компонентам тензоров упругих модулей фаз: $C_{i j k l}^{I} \ll C_{i j k l}^{f, m}$, то скачком вектора нормальных напряжений на интерфейсе можно пренебречь и соотношения (2) записываются в виде

$$
\left\{\begin{array}{l}
{\left[U_{i(p q)}^{*}\right]=\frac{1}{k_{i}} \tau_{i(p q)}^{*} ; \quad \xi \in \Sigma_{f m} ;} \\
{\left[\tau_{i(p q)}^{*}\right]=0 ; \quad \xi \in \Sigma_{f m},}
\end{array}\right.
$$

где $U_{i(p q)}^{*}, \tau_{i(p q)}^{*}-$ компоненты вектора «псевдоперемещений» и нормальных «псевдонапряжений» в системе координат интерфейса $\left\{s_{1}, s_{2}, n\right\}$ (рис.2б), $k_{i}$ - коэффициенты скачка перемещений, также называемые пружинными коэффициентами интерфейса [16]. При этом предполагается равенство коэффициентов в тангенциальном к поверхности контакта направлении: $k_{1}=k_{2}$. 
С учетом сказанного выше запишем локальную задачу с условиями мягкого неидеального интерфейса (1):

$$
\left\{\begin{array}{l}
\frac{\partial \sigma_{i j(p q)}}{\partial \xi_{i}}=0 ; \\
\varepsilon_{i j(p q)}=\frac{1}{2}\left(\frac{\partial U_{i}(p q)}{\partial \xi_{j}}+\frac{\partial U_{j}(p q)}{\partial \xi_{i}}\right) ; \\
\sigma_{i j(p q)}=C_{i j k l}^{\alpha} \varepsilon_{i j(p q)} ; \quad \xi \in V_{\alpha} ; \quad \alpha=f, m, \\
{\left[U_{i(p q)}^{*}\right]=\frac{1}{k_{i}} \tau_{i(p q)}^{*} ; \quad\left[\tau_{i(p q)}^{*}\right]=0 ; \quad \xi \in \Sigma_{f m} .}
\end{array}\right.
$$

2.1. Процедура осреднения на ячейке периодичности. Для построения тензора $\bar{C}_{i j k l}$ необходимо к компонентам тензора $\sigma_{i j(p q)}$ применить процедуру осреднения по объему ячейки периодичности. Для локальной задачи (1) эта процедура выглядела бы так:

$$
\left\langle\sigma_{i j(p q)}\right\rangle=\frac{1}{V_{f}} \int_{V_{f}} \sigma_{i j(p q)} d V+\frac{1}{V_{m}} \int_{V_{m}} \sigma_{i j(p q)} d V+\frac{1}{V_{I}} \int_{V_{I}} \sigma_{i j(p q)} d V .
$$

По принятым условиям неидеального интерфейса, поле $\sigma_{i j(p q)}$ неразрывно и осреднение по объему данного поля для задачи (4) можно просто записать как осреднение поля в двухфазном композите с условиями идеального интерфейса:

$$
\left\langle\sigma_{i j(p q)}\right\rangle=\frac{1}{V_{f}} \int_{V_{f}} \sigma_{i j(p q)} d V+\frac{1}{V_{m}} \int_{V_{m}} \sigma_{i j(p q)} d V .
$$

Отметим, что компоненты тензора $\bar{\varepsilon}_{i j}$ являются входными данными задачи (участвуют в формировании граничных условий [40]), но при этом могут быть вычислены по решениям локальных задач с помощью осреднения $\varepsilon_{i j(p q)}$. В [13] было показано, что в связи с разрывностью поля перемещений выражение для компонент осредненного тензора "псевдодеформаций“ для задачи (4) будет содержать интеграл скачка перемещений по поверхности интерфейса:

$$
\left\langle\varepsilon_{i j(p q)}\right\rangle=\frac{1}{V_{f}} \int_{V_{f}} \varepsilon_{i j(p q)} d V+\frac{1}{V_{m}} \int_{V_{m}} \varepsilon_{i j(p q)} d V+\frac{1}{2 V} \int_{\Sigma f m}\left(\left[U_{i(p q)}^{*}\right]+\left[U_{j(p q)}^{*}\right]\right) d S .
$$


2.2. Постановка задачи идентификации коэффициентов неидеального интерфейса. Полученная форма локальной задачи (4) не содержит толщины адгезионного слоя, но все еще содержит неизвестные - вектор коэффициентов скачка перемещений $\mathbf{k}=\left(k_{1}, k_{3}\right)$. Задача одного поверочного расчета композита на практике возникает крайне редко. Обычно исследуются зависимости упругих свойств как функции концентрации или некоторых параметров геометрии. Воспользуемся этим фактом.

Пусть для каждой концентрации $\phi_{j}$ из вектора концентраций наполнителя ф ставится задача отыскания эффективного вектора упругих технических констант композита $\overline{\mathbf{P}}_{\phi_{j}}=\left(\overline{\mathbf{E}}^{T}, \overline{\boldsymbol{\mu}}^{T}, \overline{\mathbf{G}}^{T}\right)$, состоящего из эффективных модулей Юнга $\bar{E}_{i}$, эффективных коэффициентов Пуассона $\bar{\mu}_{i j}$ и эффективных модулей сдвига $\bar{G}_{i j}$. Процедура построения компонент вектора $\overline{\mathbf{P}}_{i}$ по компонентам тензора $\bar{C}_{i j k l}$ описана в [33].

Пусть выбрана концентрация наполнителя $\phi_{j}$, для которой экспериментально установлен вектор технических упругих модулей композита $\mathbf{P}_{\phi_{j}}^{\exp }$, тогда коэффициенты $k_{i}$ можно найти с помощью решения задачи оптимизации вида

$$
\min _{k}\left\|H_{\phi_{j}}(\mathbf{k})-\mathbf{P}_{\phi_{j}}^{\exp }\right\|
$$

где $H_{\phi_{j}}()$ - функция задачи гомогенизации, вычисляющая вектор $\overline{\mathbf{P}}_{\phi_{j}}$. Каждый вектор $\overline{\mathbf{P}}_{\phi_{j}}$ для соответствующей концентрации $\phi_{j}$ может быть найден с использованием ранее полученных коэффициентов $k_{i}$, определяющих скачки поля перемещений.

Обширное исследование по решению задач идентификации упругих констант межфазного слоя с использованием трехфазной модели можно найти в предыдущей работе авторов [41]. Общие подходы к регуляризации и анализу устойчивости задач вида (8), изложенные в данной работе, применимы к задаче идентификации коэффициентов неидеального интерфейса.

\section{3. Конечно-элементное решение локальных задач со скачками поля пе- ремещения}

Будем решать задачи (4) с помощью метода конечных элементов в перемещениях. Для дискретизации объемной расчетной области компонент воспользуемся распространенным тетраэдальным конечным элементом с линейными функциями формы. Подробное описание решения локальных задач с помощью данных конечных элементов можно найти в [33]. В конеч- 
но-элементную модель задачи $L_{p q}$ необходимо добавить соотношения (3), связывающие поля перемещений на поверхности фаз. Для этого введем специальный интерфейсный поверхностный конечный элемент.

Схема конечного элемента представлена на рис.3. Элемент состоит из двух треугольников, один из которых принадлежит тетраэдру фазы $f$ (построен на тех же узлах), второй - фазе $m$. Расстояние $D$ между треугольниками до деформации равно нулю.

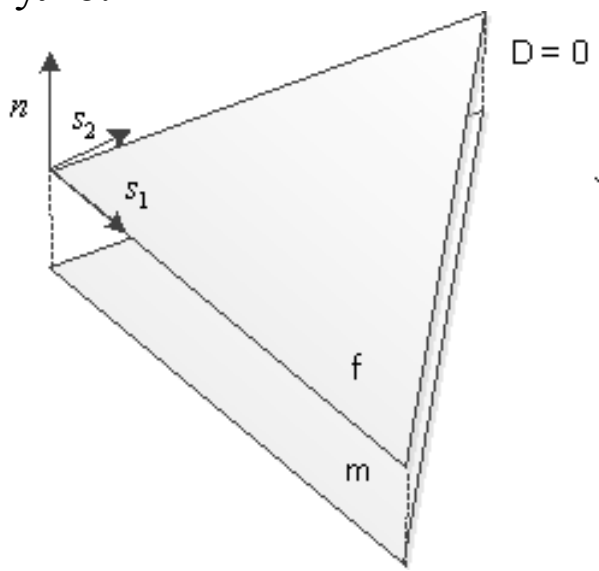

Рис.3. Схема поверхностного интерфейсного конечного элемента.

Построим процедуру получения локальной матрицы жесткости такого элемента. Для каждого треугольника поле перемещений в локальной системе координат может быть представлено в виде:

$$
\mathbf{U}^{*}=\left[N_{1}[I], N_{2}[I], N_{3}[I]\right]\left\{\begin{array}{l}
\{U\}_{1}^{*} \\
\{U\}_{2}^{*} \\
\{U\}_{3}^{*}
\end{array}\right\}=[N]\{U\}_{\text {nodal }}^{*},
$$

где $N_{i}$ - функция формы $i$-го узла; $[I]-$ единичная матрица $3 \times 3 ;\{U\}_{i}^{*}-$ вектор перемещений в $i$-м узле; $[N]$ и $\{U\}_{\text {nodal }}^{*}$ - матрица функций формы и вектор узловых перемещений треугольного элемента. Тогда поля перемещений в треугольном элементе наполнителя и матрицы равны соответственно:

$$
\begin{aligned}
& \mathbf{U}^{* f}=[N]^{f}\{U\}_{\text {nodal }}^{* f}, \\
& \mathbf{U}^{* m}=[N]^{m}\{U\}_{\text {nodal }}^{{ }^{* m}} .
\end{aligned}
$$

Согласно условиям неидеального интерфейса, поле перемещений между треугольными элементами должно претерпевать разрыв:

$$
\left[\mathbf{U}^{*}\right]=\mathbf{U}^{* f}-\mathbf{U}^{* m}=\left[[N]^{f},-[N]^{m}\right]\left\{\begin{array}{l}
\{U\}_{\text {nodal }}^{* f} \\
\{U\}_{\text {nodal }}^{{ }^{*} m}
\end{array}\right\} .
$$


Приведем данное выражение к каноническому для МКЭ виду:

$$
\left[\mathbf{U}^{*}\right]=[B]\{U\}_{\text {nodal }}^{* e},
$$

где $\{U\}_{\text {nodal }}^{* e}$ - вектор узловых перемещений интерфейсного элемента, $[B]-$ матрица, состоящая из матриц функций форм треугольных элементов наполнителя и матрицы:

$$
[B]=\left[[N]^{f},-[N]^{m}\right] .
$$

В конечно-элементном виде соотношения неидеального интерфейса (3) для векторов нормальных напряжений в локальной системе координат элемента могут быть записаны так:

$$
\boldsymbol{\tau}^{* f}=\boldsymbol{\tau}^{* m}=[k][B]\{U\}_{\text {nodal }}^{*_{e}},
$$

где $[k]$ - матрица коэффициентов неидеального интерфейса:

$$
[k]=\left[\begin{array}{lll}
k_{1} & 0 & 0 \\
0 & k_{2} & 0 \\
0 & 0 & k_{3}
\end{array}\right]=\left[\begin{array}{lll}
k_{s} & 0 & 0 \\
0 & k_{s} & 0 \\
0 & 0 & k_{n}
\end{array}\right] .
$$

Матрицу жесткости такого элемента в локальной системе координат находим с помощью интегрирования по площади треугольника $A$ :

$$
[K]^{* e}=\int_{A}[B]^{T}[k][B] d A .
$$

Построение матрицы жесткости в глобальной системе координат $[K]^{e}$ производим с помощью стандартной процедуры смены базиса оператора:

$$
\begin{aligned}
{[K]^{e} } & =[\theta]^{T}[K]^{* e}[\theta], \\
{[\theta] } & =\left[\begin{array}{cccccc}
{[\lambda]^{T}} & \mathbf{O} & \mathbf{O} & \mathbf{O} & \mathbf{O} & \mathbf{O} \\
\mathbf{O} & {[\lambda]^{T}} & \mathbf{O} & \mathbf{O} & \mathbf{O} & \mathbf{O} \\
\mathbf{O} & \mathbf{O} & {[\lambda]^{T}} & \mathbf{O} & \mathbf{O} & \mathbf{O} \\
\mathbf{O} & \mathbf{O} & \mathbf{O} & {[\lambda]^{T}} & \mathbf{O} & \mathbf{O} \\
\mathbf{O} & \mathbf{O} & \mathbf{O} & \mathbf{O} & {[\lambda]^{T}} & \mathbf{O} \\
\mathbf{O} & \mathbf{O} & \mathbf{O} & \mathbf{O} & \mathbf{O} & {[\lambda]^{T}}
\end{array}\right],
\end{aligned}
$$

где $[\lambda]=\left[\{s 1\}^{T},\{s 2\}^{T},\{n\}^{T}\right]-$ матрица перехода от системы координат элемента к глобальной системе координат; О - нулевая матрица $3 \times 3$. 
Для процедуры осреднения (7) необходимо вычислить интеграл скачка перемещений по площади элемента:

$$
\int_{A}\left[\mathbf{U}^{*}\right] d A=\int_{A}[B]\left(\{U\}_{\text {nodal }}^{* f}-\{U\}_{\text {nodal }}^{*_{m}}\right) d A .
$$

В выражениях (17), (20) компоненты матрицы $[B]$ являются функциями координат. Для численного интегрирования используем естественные координаты и квадратуры Гаусса-Радо [42].

Преимуществами представленного элемента является возможность построить локальную систему координат по расчетной сетке. Но при этом необходимо договориться о выборе направления для нормали $n$, так как от него зависит знак скачка поля перемещений. К примеру, можно условиться, что нормаль направлена от фазы $f$ к фазе $m$.

\section{4. Численное сравнение трёхфазной модели и модели неидеального ин- терфейса}

В $[14,16]$ для дисперсно-армированного сферическими включениями и однонаправленного композита выведены зависимости между упругими характеристиками (модуль Юнга $E_{i}$, коэффициент Пуассона $\mu_{i}$ ) и толщиной $D$ объемного межфазного слоя трехфазной модели и коэффициентами модели мягкого неидеального интерфейса.

Используя эти соотношения, сравним результаты расчетов эффективных упругих характеристик с использованием трехфазной модели (1) и модели неидеального интерфейса (4). Расчеты проведем для изотропного дисперсно-армированного стеклянными сферами композита из [43] и трансверсально-изотропного однонаправленного композита (рис.1а), армированного стеклянными волокнами из [44]. Рассматриваем композит с концентрацией наполнителя $\phi_{j}=30 \%$.

Введем обозначение $\eta$ для отношения толщины межфазного слоя к характерному размеру наполнителя. В случае дисперсно-армированного композита расчеты проводим при значениях $\eta=0.01,0.001$. На рис.4, 5 представлены зависимости эффективных характеристик композита: модуля Юнга $E_{c}$ и коэффициента Пуассона $\mu_{c}$ от модуля Юнга межфазного слоя $E_{i}$. Характеристики отнесены к упругим свойствам матрицы $E_{m}$ и $\mu_{m}$. Коэффициент Пуассона межфазного слоя в данном случае является константой, равной $\mu_{i}=0.49$, что в соответствии с [4] является адекватной оценкой для композитов с полимерной матрицей. 
Можно отметить, что для $\eta=0.01$ результаты расчета модуля Юнга композита с разными моделями интерфейса совпадают, если модуль Юнга матрицы не менее чем на порядок превосходит модуль Юнга межфазного слоя. Для расчета коэффициента Пуассона данные модели дают эквивалентный результат, если модуль Юнга матрицы превосходит модуль Юнга межфазного слоя не менее чем на порядок, но не более чем на 4 порядка. Для $\eta=0.001$ расчеты показывают эквивалентность моделей, если модуль Юнга межфазного слоя не превосходит модуль Юнга матрицы на 3 порядка. Эти результаты соответствуют теоретическому исследованию [13].
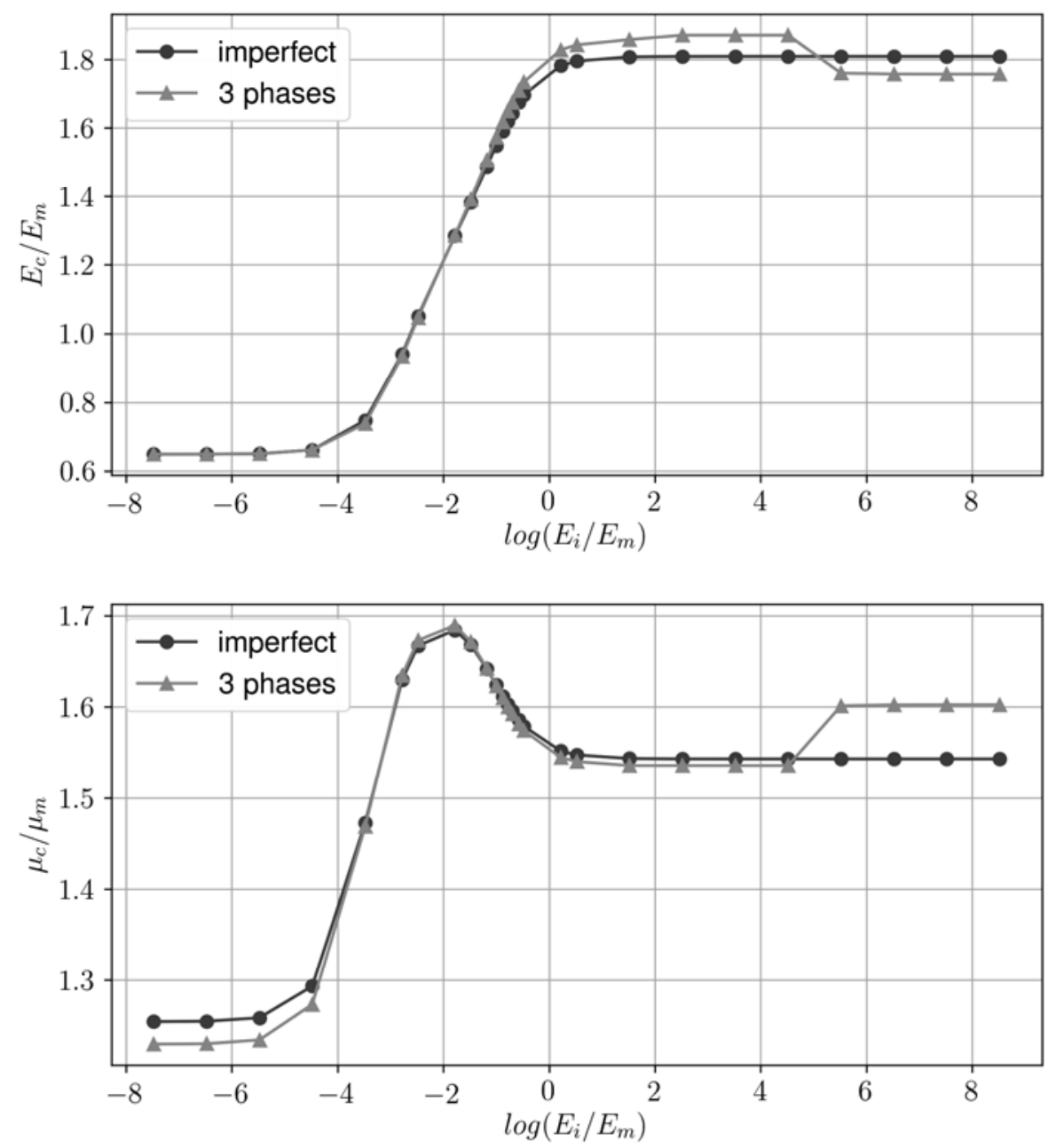

Рис.4. Сравнение результатов расчета эффективных упругих свойств изотропного композита для $\eta=0.01$.

Для трансверсально-изотропного композита сравнение проводилось при $\eta=0.001$. По представленным на рис. 6 зависимостям можно сказать, что модели дают эквивалентный результат, если модуль Юнга матрицы превосходит модуль Юнга межфазного слоя не менее чем на порядок, но не более чем на 4 порядка. 

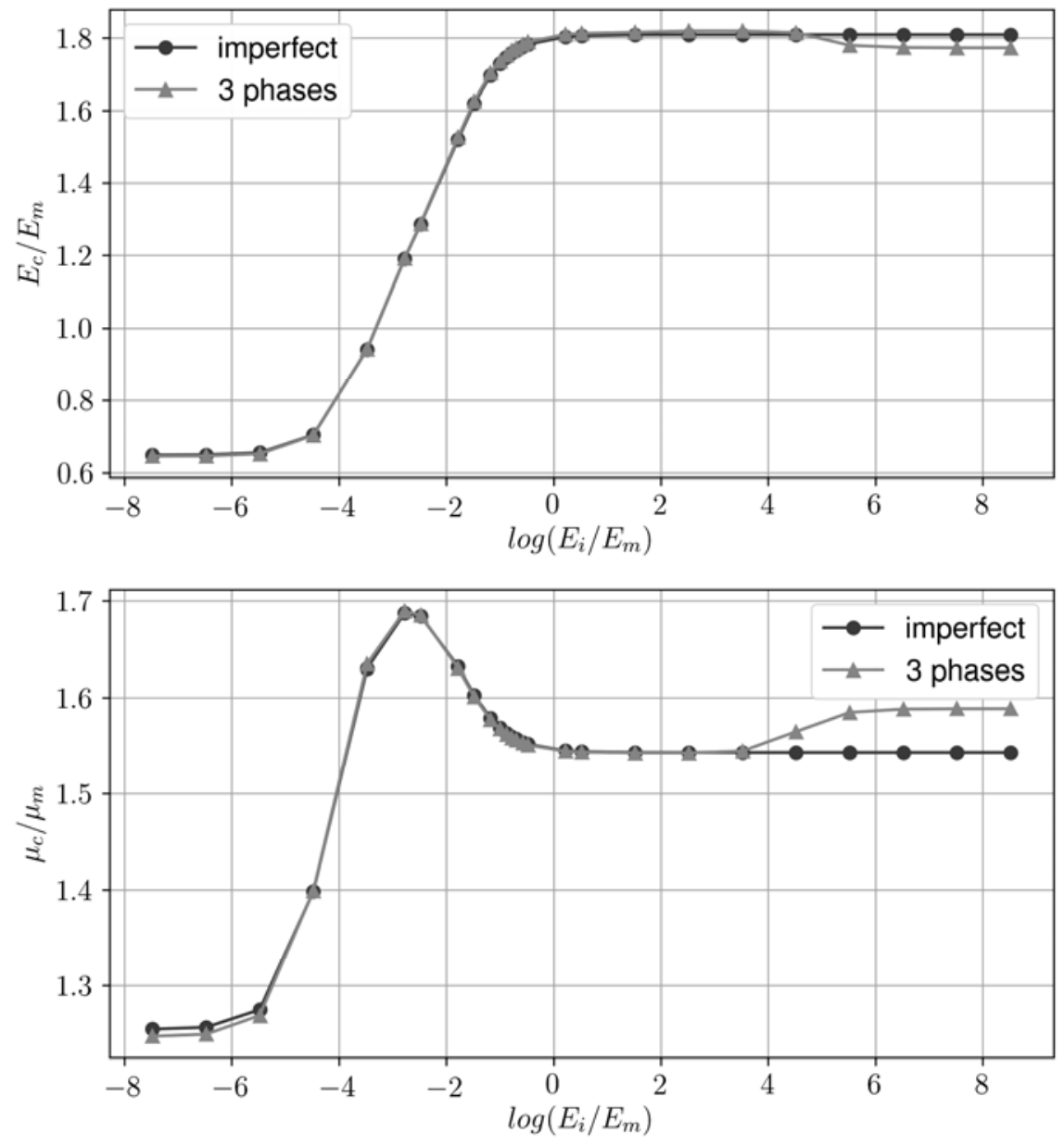

Рис.5. Сравнение результатов расчета эффективных упругих свойств изотропного композита для $\eta=0.001$.

\section{5. Численная идентификация коэффициентов неидеального интерфейса}

Для дисперсно-армированного стеклянными сферами композита из работы [43] поставим задачу численной идентификации коэффициентов неидеального интерфейса $\mathbf{k}$ по экспериментально полученным модулю Юнга $E_{c}^{e x p}$ и коэффициенту Пуассона $\mu_{c}^{\text {exp }}$ для концентрации $\phi_{j}=30 \%$ :

$$
\min _{\mathbf{k}^{*}}\left(\left(\frac{E_{c}(k)-E_{c}^{\exp }}{E_{c}^{\exp }}\right)^{2}+\left(\frac{\mu_{c}(k)-\mu_{c}^{\exp }}{\mu_{c}^{\exp }}\right)^{2}\right),
$$

где $E_{c}, \mu_{c}$ - эффективные упругие константы, вычисленные с помощью численного метода асимптотического осреднения с условиями неидеального интерфейса, описанного в данной работе. Задачу оптимизации (21) решаем с помощью метода последовательного квадратичного программирования. По полученным $\mathbf{k}^{*}$ вычисляем упругие технические константы для других концентраций и сравниваем с экспериментальными данными из работы [43] и упругими техническими константами, рассчитанными с использованием модели идеального интерфейса (рис.7). 

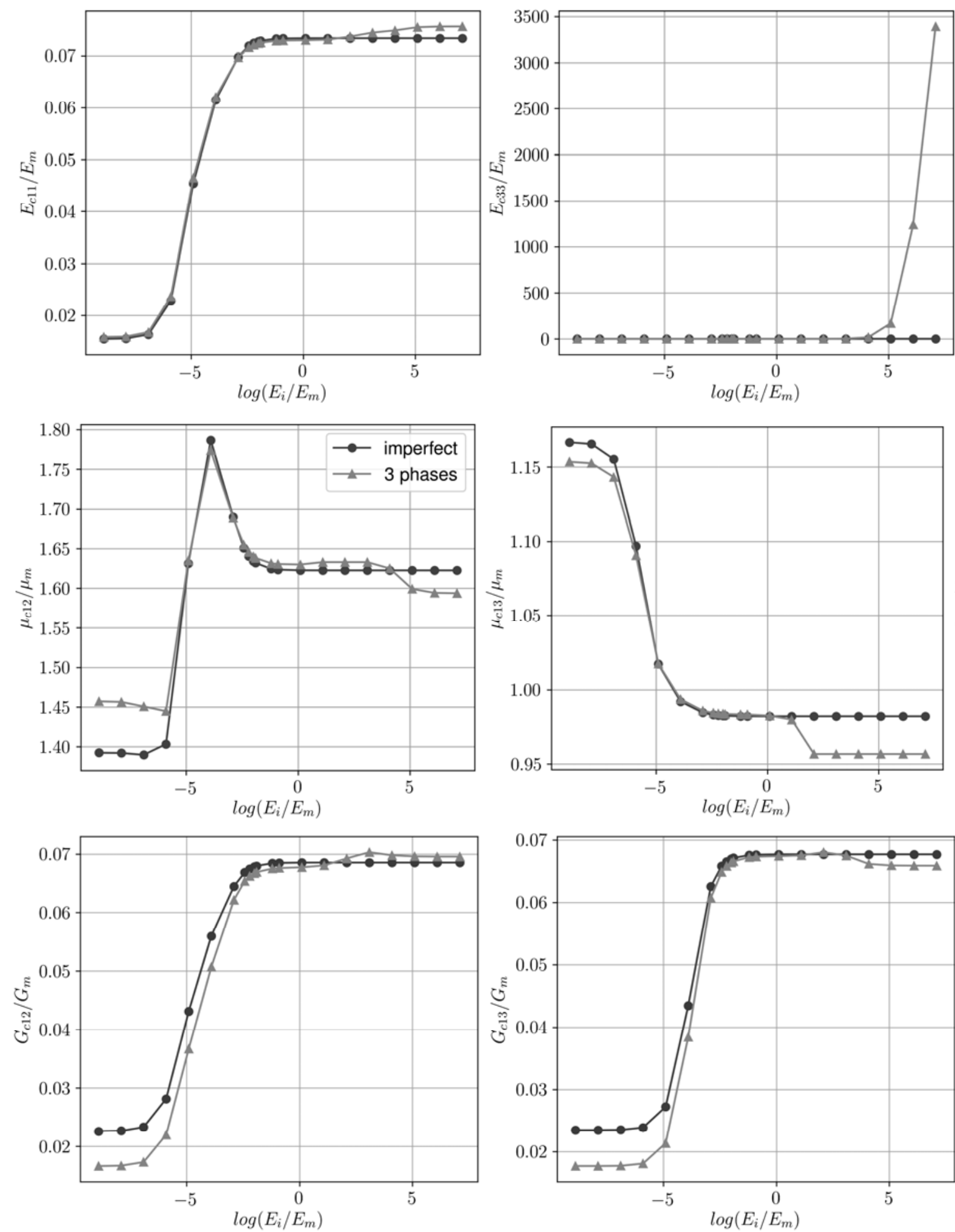

Рис.6. Сравнение результатов расчета эффективных упругих свойств трансверсально-изотропного композита для $\eta=0.001$ (продолжение).

По представленным зависимостям можно сказать следующее:

- Для концентраций наполнителя больше $20 \%$ упругие технические константы, рассчитанные с условиями идеального интерфейса между фазами, обладают существенной погрешностью по сравнению с экспериментом. 
- Введение условий неидеального интерфейса с коэффициентами, идентифицированными по экспериментальным данным для одной концентрации, позволяют существенно повысить точность расчета для композитов с высокой концентрацией наполнителя.
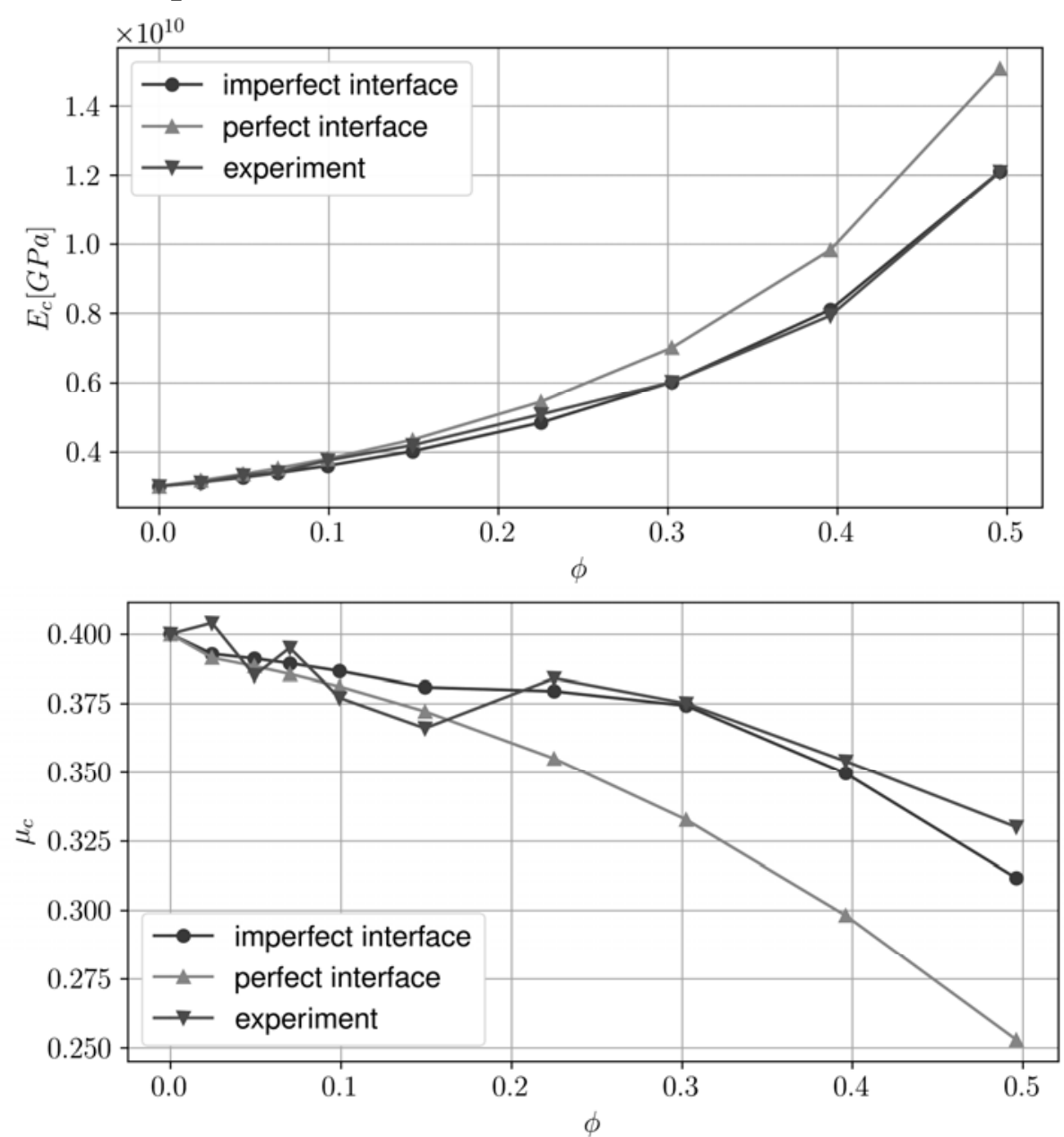

Рис.7. Сравнение с экспериментальными данными упругих свойств композита, рассчитанных в условиях идеального и неидеального интерфейса.

\section{6. Заключение}

В результате исследования разработана модификация численного метода асимптотического осреднения для расчета эффективных упругих свойств композита с условиями неидеального интерфейса. Для реализации метода разработана математическая модель поверхностного интерфейсного элемента. Вычислительные эксперименты с применением реализованного метода показывают, что

- в широком диапазоне значений отношения жесткости межфазного слоя к жесткости матрицы, результаты решения задачи гомогенизации упругих свойств с условиями неидеального интерфейса эквиваленты результатам расчета с помощью трехфазной модели;

- ширина диапазона, в котором модели эквивалентны, зависит от отно- 
шения толщины межфазного слоя к характерному размеру наполнителя и схемы армирования;

- возможна идентификация коэффициентов неидеального интерфейса по экспериментально полученным техническим константам композита для одной концентрации;

- с помощью идентифицированных коэффициентов можно предсказывать упругие характеристики более точно для композитов с высокой концентрацией наполнителя.

Резюмируя сказанное выше, можно отметить, что в работе представлено математическое обоснование и техническая реализация комплексного подхода для расчета упругих характеристик композита с условиями мягкого неидеального интерфейса. Для ряда композитов численно определены границы его применимости.

\section{СПИСОК ЛИТЕРАТУРЫ}

1. J.D. Achenbach, H. Zhu. Effect of interfacial zone on mechanical behavior and failure of fiber-reinforced composites // J. of the Mech. and Phys. of Sol., 1989, v.37, №3, p.81-393.

2. В.Э. Згаевский, Ю.Г. Яновский. Механические характеристики слоя макромолекул вблизи поверхности наполнителя // Мех. композ. матер. и конструк., 1997, т.3, № 1, с.105-112; V.E. Zgaevskij, IU.G. IAnovskij. Mekhanicheskie kharakteristiki sloia makromolekul vblizi poverkhnosti napolnitelia // Mekh. kompozits. mater. i konstruk., 1997, t.3, №1, s.105-112.

3. P.G. Khalatur. Computer simulation of thin polymer layers // Makromol. Chem., Macromol. Symp., 1991, v.44, № 1, p.23-32.

4. Н.К. Балабаев, А.Н. Власов, В.Э. Згаевский, Ю.Н. Карнет, Ю.Г. Яновский. Структура и микромеханические свойства межфазных слоёв полимерных матричных композитов // Механика композиционных материалов и конструкций, 1999, т.5, № 2, с.109-123;

N.K. Balabaev, A.N. Vlasov, V.E. Zgaevskij, IU.N. Karnet, IU. G. IAnovskij. Struktura i mikromekhanicheskie svojstva mezhfaznykh sloev polimernykh matrichnykh kompozitov // Mekhanika kompozitsionnykh materialov i konstruktsij, 1999, t.5, №2, s.109-123.

5. R.M. Christensen, K.H. Lo. Solutions for effective shear properties in three phase sphere and cylinder models // J. of the Mechanics and Physics of Solids, 1979, v.27, №4, p.315-330.

6. Yozo Mikata, Minoru Taya. Stress field in a coated continuous fiber composite subjected to thermomechanical loadings // J. Compos. Mater., November, 1985, v.19, № 6, p.554-578.

7. Y. Benveniste, G.J. Dvorak, T. Chen. Stress fields in composites with coated inclusions // Mechanics of Materials, 1989, v.7, №4, p.305-317.

8. N.J. Pagano, G.P. Tandon. Elastic response of multi-directional coated-fiber composites. Composites Science and Technology, 1988, v.31, № 4, p.273-293.

9. I. Sevostianov, R. Rodriguez-Ramos, R. Guinovart-Diaz, J. Bravo-Castillero, F.J. Sabina. Connections between different models describing imperfect interfaces in periodic fiberreinforced composites // Intern. J. of Solids and Structures, 2012, v.49, №13, p.1518-1525.

10. M. Goland, E. Reissner. The stresses in cemented joints // J. Appl. Mech., 1944, v.66, p.A17-A27. 
11. Y. Benveniste. The effective mechanical behaviour of composite materials with imperfect contact between the constituents // Mechanics of Materials, 1985, v.4, №2, p.197-208.

12. Z. Hashin. Thermoelastic properties of fiber composites with imperfect interface // Mechanics of Materials, 1990, v.8, №4, p.333-348.

13. Z. Hashin. The spherical inclusion with imperfect interface // J. of Applied Mechanics, 1991, v.58, №2, p.444-449.

14. Z. Hashin. Thermoelastic properties of particulate composites with imperfect interface // Journal of the Mechanics and Physics of Solids, 1991, v.39, №6, p.745-762.

15. Z. Hashin. Extremum principles for elastic heterogeneous media with imperfect interfaces and their application to bounding of effective moduli // J. of the Mechanics and Physics of Solids, 1992, v.40, №4, p.767-781.

16. Z. Hashin. Thin interphase/imperfect interface in elasticity with application to coated fiber composites // J. of the Mechanics and Physics of Solids, 2002, v.50, №12, p.2509-2537.

17. P. Bövik. On the modelling of thin interface layers in elastic and acoustic scattering problems // Quarterly J. of Mechanics and Appl. Math., February 1994, v.47, №1, p.17-42.

18. Y. Benveniste, T. Miloh. Imperfect soft and stiff interfaces in two-dimensional elasticity // Mechanics of Materials, 2001, v.33, №6, p.309-323.

19. D. Caillerie, J.C. Nedelec. The effect of a thin inclusion of high rigidity in an elastic body // Mathematical Methods in the Applied Sciences, 1980, v.2, №3, p.251-270.

20. Y. Benveniste. A general interface model for a three-dimensional curved thin anisotropic interphase between two anisotropic media // J. of the Mechanics and Physics of Solids, 2006, v.54, № 4, p.708-734.

21. A. Klarbring. Derivation of a model of adhesively bonded joints by the asymptotic expansion method // Intern. J. of Engineering Science, 1991, v.29, №4, p.493-512.

22. A. Klarbring. A.B. Movchan. Asymptotic modelling of adhesive joints // Mechanics of Materials, 1998, v.28, №1-4, p.137-145.

23. G. Geymonat, F. Krasucki, S. Lenci. Mathematical analysis of a bonded joint with a soft thin adhesive // Mathematics and Mechanics of Solids, 1999, v.4, №2, p.201-225.

24. F. Lebon, R. Rizzoni, S. Ronel, C. Licht. Analysis of non-linear soft thin interfaces // The Sixth Intern. Conf. on Comp. Structures Technology, September 2002, v.82, p.155-156.

25. F. Lebon, S. Ronel. Asymptotic analysis of Mohr-Coulomb and Drucker-Prager soft thin layers // Steel and Composite Structures, Techno-press, 2004, v.4, p.133-147.

26. F. Lebon, R. Rizzoni. Asymptotic behavior of a hard thin linear elastic interphase: an energy approach // Intern. J. of Solids and Structures, Elsevier, 2011, v.48, №3-4, p.441-449.

27. R. Rizzoni, S. Dumont, F. Lebon, E. Sacco. Higher order model for soft and hard elastic interfaces // Intern. J. of Solids and Structures, 2014, v.51, №23-24, p.4137-4148.

28. Н.С. Бахвалов, Г.П. Панасенко. Осреднение процессов в периодических средах. Математические задачи механики композиционных материалов. - М.: Наука, 1984.

N.S. Bakhvalov, G.P. Panasenko. Homogenization: averaging processes in periodic media. Mathematical problems in mechanics of composite materials. Ser. Mathematics and its applications, Dordrecht, Boston, London: Kluwer academic publishers, 1989, v.36, 34p.

29. Б.Е. Победря. Механика композиционных материалов. - М.: МГУ, 1984.

B.E. Pobedria. Mekhanika kompozitsionnykh materialov [Mechanics of composite materials]. - M.: MGU, 1984. 
30. K.A. Wilkinson, D.A. Ordonez. Adhesive properties in nanomaterials // Nova Science Publishers, Inc., 2011, 188 p.

31. F. Lebon, S. Dumont, R. Rizzoni, J.C. López-Realpozo, R. Guinovart-Díaz, R. RodríguezRamos, J. Bravo-Castillero, F.J. Sabina. Soft and hard anisotropic interface in composite materials // Composites Part B: Engineering, Elsevier, 2016, v.90, p.58-68.

32. R. Rodríguez-Ramos, R. de Medeiros, R. Guinovart-Díaz, J. Bravo-Castillero, J.A. Otero, V. Tita. Different approaches for calculating the effective elastic properties in composite materials under imperfect contact adherence // Composite Struct., Elsevier, 2013, v.99, p.264-275.

33. Y.I. Dimitrienko, A.P. Sokolov. Elastic properties of composite materials // Mathematical Models and Computer Simulations, 2010, v.2, №1, p.116-130.

34. Ю.И. Димитриенко, А.П. Соколов. Численное моделирование композиционных материалов с многоуровневой структурой // Изв. РАН. Физ. сер., 2011, т.75, №11, с.1551-1556.

IU.I. Dimitrienko, A.P. Sokolov. CHislennoe modelirovanie kompozitsionnykh materialov s mnogourovnevoj strukturoj // Izv. RAN. Fizicheskaia ser., 2011, t.75, №11, s.1551-1556.

35. P. Rahul-Kumar, A. Jagota, S.J. Bennison, S. Saigal, S. Muralidhar. Polymer interfacial fracture simulations using cohesive elements // Acta Materialia, 1999, v.47, №15-16, p.4161-4169.

36. W.G. Jiang, R.Z. Zhong, Q.H. Qin, Y.G. Tong. Homogenized finite element analysis n effective elastoplastic mechanical behaviors of composite with imperfect interfaces // Intern. Journal of Molecular Sciences, 2014, v.15, №12, p.23389-23407.

37. S. Guessasma, N. Benseddiq, D. Lourdin. Effective Young's modulus of biopolymer composites with imperfect interface // Int. J. of Solids and Struct., Elsev., 2010, v.47, №18-19, p.2436-2444.

38. A.A. Nasedkina, A. Rajagopal. Mathematical and computer homogenization models for bulk mixture composite materials with imperfect interfaces // Materials Physics and Mechanics, 2018, v.37, №1, p.31-34.

39. M. Hamsasew, W.S. Yu. A micromechanical approach to imperfect interface analysis of heterogeneous materials // 56th AIAA/ASCE/AHS/ASC Structures, Structural Dynamics, and Materials Conference, 2015.

40. V. Schetinin, Kh. Dmitriy. nla3d - open source FEM framework. url: https://github. com/ dmitryikh/nla3d/, 2018.

41. А.П. Соколов, В.Н. Щетинин. Идентификация упругих свойств адгезионного слоя дисперсно-армированных композитных материалов на основе экспериментальных данных // Механика композиционных материалов и конструк., 2018, т.24, №4, с.555-581. A.P. Sokolov, V.N. Shchetinin. Identifikatsiia uprugikh svojstv adgezionnogo sloia dispersno-armirovannykh kompozitnykh materialov na osnove eksperimentalnykh dannykh // Mekhanika kompozitsionnykh materialov i konstruktsij, 2018, t.24, №4, s.555-581.

42. O.C. Zienkiewicz. The finite element method in engineering science. - London; New York: McGraw-Hill, 1971, $521 \mathrm{p}$.

43. J. Smith. Experimental values for the elastic constants of a particulate filled glassy polymer // J. Res. NBS., 1976, v.80A, №1, p.45-49.

44. P.D. Soden, M.J. Hinton, A.S. Kaddour. Lamina properties, lay-up configurations and loading conditions for a range of fibre-reinforced composite laminates // Composites Science and Technology, 1998, v.58, №7, p.1011-1022. 